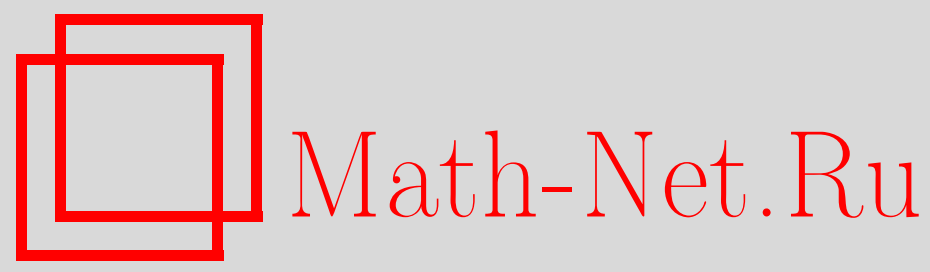

Е. В. Радкевич, О глобальной устойчивости решений систем моментов неравновесной термодинамики, Матем. заметки, 2003, том 73, выпуск 4, 590-602

DOI: https://doi.org/10.4213/mzm207

Использование Общероссийского математического портала Math-Net.Ru подразумевает, что вы прочитали и согласны с пользовательским соглашением http://www.mathnet.ru/rus/agreement

Параметры загрузки:

IP : 34.229 .108 .108

26 апреля 2023 г., $07: 13: 17$

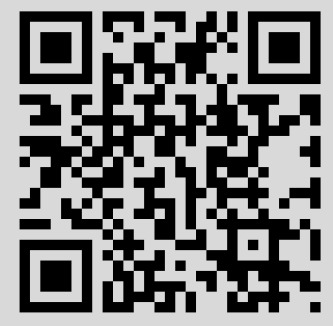




\section{О ГЛОБАЛЬНОЙ УСТОЙЧИВОСТИ РЕШЕНИЙ СИСТЕМ МОМЕНТОВ НЕРАВНОВЕСНОЙ ТЕРМОДИНАМИКИ}

\section{Е.В. Радкевич}

В статье исследуются линеаризации в окрестности состояния равновесия задачи Коши и смешанной задачи для систем моментов Града-Эрмита неравновесной термодинамики. Получены условия устойчивости решений задачи Коши как обобщение классической теоремы Эрмита-Биллера об устойчивых полиномах. Для смешанной задачи получен аналог теоремы Вишика-Люстерника о малых сингулярных возмущениях общих эллиптических задач. Последнее позволило определить условие Шапиро-Лопатинского о корректности смешанной задачи.

Библиография: 12 названий.

1. Введение. Целью этой статьи является исследование задачи Коши и смешанной задачи для систем моментов Града-Эрмита, полученных в контексте неравновесной термодинамики [1]-[3], базирующейся на кинетической теории одно-атомного идеального газа. Определяя термодинамические величины - массу, энергию, импульс, энтропию и т.д. - как моменты функции распределения, кинетика сводит исследование кинетических уравнений к бесконечной системе квазилинейных уравнений с частными производными - законов сохранения с релаксацией. Существует множество способов замыкания этой системы (обрыва бесконечной цепочки уравнений), один из которых метод Эрмита-Града, позволяющих аппроксимировать эту систему конечной системой $N$ законов сохранения, имеющих дважды диввергентную форму относительно первых $N$ моментов. Любой принцип замыкания бесконечной цепочки моментных уравнений опирается на предположение, что по крайней мере для малых начальных (начально-краевых в случае смешанной задачи) отклонений от состояния равновесия решения полученных систем законов сохранения существуют глобально; более того, эти решения близки к решению кинетического уравнения. Имеется в виду, что при подстановке решения кинетического уравнения (функции распределения) в замыкание моментной системы мы получим разумно малые невязки. Недавно в [4] для системы моментов, полученной замыканием методом максимальной энтропии для кинетического уравнения Ловермо (уравнения типа Больцмана), было показано, что область допустимых значений моментов является невьпуклой. Более того, она имеет внутреннюю границу, содержашую все равновесные состояния, в которых старший момент имеет степенную особенность. Заметим, что подобного рода особеность не возникает при замыкании методом Эрмита-Града. Невыпуклость области допустимых значений в окрестности состояний равновесия нарушает основное условие глобальной разрешимости гиперболических симметризуемых систем [5]. Поэтому важнейшей задачей, требующей решения в связи с результатами [4], 
является выяснение природы сингулярности метода максимальной энтропии. Вызвана ли невьпуклость области допустимых значений в окрестности состояний равновесия самим методом замыкания или отражает свойства решений кинетического уравнения? Хорошо известно, что иерархия систем моментов содержит систему уравнений газовой динамики, полученную феномологическим замыканием кинетических уравнений законами Фурье-Навье-Стокса. Возможно, что природа трудностей с доказательством их глобальной разрешимости связана с невьпуклостью области допустимых значений для систем моментов - высших по иерархии.

Результаты [4] показали, что необходимо дополнительное исследование, казалось так хорошо известных, линеаризованных систем моментов в окрестности состояния равновесия. В этой статье мы приведем анализ линеаризованных систем моментов ГрадаЭрмита, который показал, что они обладают чрезвычайно жесткой структурой: дисперсионные уравнения задачи Коши для этих систем являются гиперболическими пучками, описьваемыми строгими и нестрогими цепочками гиперболических полиномов [6].

Линеаризованная в окрестности состояния равновесия $\varrho=\varrho_{E}, v_{i}=0, T=T_{E}$, $p_{\langle i j\rangle}=0, q_{i}=0, \varrho_{\langle i j k\rangle}=0, \varrho_{k k\langle i j\rangle}=0$ 26-моментная система [1] имеет следуюший вид:

$$
\begin{gathered}
\partial_{t} \varrho+\partial_{x_{k}} v_{k}=0 \\
\partial_{t} v_{i}+\frac{3}{5}\left(\partial_{x_{i}} \varrho+R \partial_{x_{k}} T\right)+\partial_{x_{k}} p_{\langle i k\rangle}=0 \\
R \partial_{t} T-\frac{2}{5} \partial_{t} \varrho+\frac{2}{3} \partial_{k} q_{k}=0, \\
\partial_{t} p_{\langle i j\rangle}+\frac{4}{5} \frac{\partial q_{\langle i}}{\partial_{\left.x_{j}\right\rangle}}+\partial_{x_{k}} \varrho_{\langle i j k\rangle}+\frac{6}{5} \frac{\partial v_{\langle i}}{\partial_{\left.x_{j}\right\rangle}}=-B p_{\langle i j\rangle}, \\
\partial_{t} q_{i}+\frac{3}{5} \partial_{x_{k}} p_{\langle i k\rangle}+\frac{1}{6} \partial_{x_{i}} \Delta+\frac{1}{2} \partial_{x_{k}} \varrho_{j j\langle i k\rangle}+\frac{3}{2} R \partial_{x_{i}} T=-\frac{2}{3} B q_{i}, \\
\left.-\frac{1}{5}\left(\partial_{x_{n}} \varrho_{r r\langle i n\rangle} \delta_{j k}+\partial_{x_{n}} \varrho_{r r\langle j n\rangle} \delta_{i k}+\partial_{x_{n}} \varrho_{r r\langle k n\rangle} \delta_{i j}\right)\right\}=-\frac{3}{2} B \varrho_{i j k}, \\
\partial_{x_{k}} \varrho_{r r\langle i j\rangle}+\frac{24}{5} \partial_{x_{k}} \varrho_{r r} q_{k}=-\frac{2}{3} B \Delta, \\
\partial_{t} \varrho_{r r\langle i j\rangle}+\frac{126}{25} \frac{\partial v_{\langle i}}{\partial_{\left.x_{j}\right\rangle}}+\frac{268}{25} \frac{\partial q_{\langle i}}{\partial_{\left.x_{j}\right\rangle}}+\frac{27}{5} \partial_{x_{k}} \varrho_{\langle i j k\rangle}=-\frac{7}{6} B\left(\varrho_{r r\langle i j\rangle}-\frac{3}{5} p_{\langle i j\rangle}\right) .
\end{gathered}
$$

Неизвестные функции: плотность $\varrho$, скорость $v_{i}$, температура $T$ и 21 неравновесных величин: $p_{\langle i j\rangle}, q_{i}, \varrho_{\langle i j k\rangle}, \varrho_{r r\langle i j\rangle}$, описьвающих отклонение от состояния равновесия. Угловые скобки в индексах обозначают симметрическую безследовую часть тензоров. Здесь $R$ - газовая постоянная, число Кнудсена $B$ является большим для сильно разряженных газов [1]. Подставляя в (1)-(8) решение типа плоских волн

$$
U=\mathcal{R} \exp (i(k x+\omega t)), \quad \mathcal{R}=\left(R^{\varrho}, R^{v_{i}}, R^{T}, R^{\left.p_{\langle i j\rangle}, R^{q_{i}}, R^{\varrho\langle i j k\rangle}, R^{\Delta}, R^{\varrho_{r r}\langle i j\rangle}\right),}\right.
$$

получаем дисперсионные уравнения для 13-, 14-, 20-, 21-, 26-моментных систем в 1-D, $2-\mathrm{D}$ и $3-\mathrm{D}$ случаях. Так, например, 13-моментная система отвечает первым 13 уравнениям в системе (1)-(8), в которых величины $\varrho_{\langle i j k\rangle}=\Delta=\varrho_{r r\langle i j\rangle}=0$; соответственно 20-моментной системе отвечают первые 20 уравнений системы (1)-(8), в которых 
$\Delta=\varrho_{r r\langle i j\rangle}=0$. Удивительным фактом является то, что все дисперсионные уравнения являются строго или нестрого гиперболическими пучками вида

$$
\mathcal{P}=\sum_{j=0}^{N}(-i)^{j} B^{j} P_{j}(\omega, k), \quad k \in \mathbb{R}^{d}, \quad d=1,2,3 .
$$

Следуя [6], приведем определение гиперболического пучка.

ОПРЕДЕЛЕНИЕ 1.1. Цепочка однородных полиномов с вещественными коэффициентами $P_{j}(\omega, k), j=0,1, \ldots, N$, порядка $l-j, l>N$, соответственно, назьвается строго зиперболическим пучком длины $N+1$, если все полиномы $P_{j}$ строго гиперболические относительно $\omega$ и корни соседних полиномов $P_{j}, P_{j+1}, j=0, \ldots, N-1$, строго взаимно разделяют друг друга, так что между каждыми двумя корнями полинома $P_{j}$ есть один корень полинома $P_{j+1}$. В случае нестрогой гиперболичности однородные полиномы цепочки нестрого гиперболические относительно $\omega$ и нестрого выполнено условие взаимного разделения корней соседних пар полиномов из цепочки.

Так, например, в двумерном случае (решение (1)-(8) зависит только от двух пространственных переменных $\left.\left(x_{1}, x_{2}\right)\right)$ дисперсионное уравнение задачи Коши для 13-моментной системы является цепочкой из шести нестрого гиперболических полиномов

$$
\begin{aligned}
& P_{0}(\omega, k)=|k|^{9} \tau^{3}\left[\tau^{6}-\frac{103}{25} \tau^{4}+\frac{21}{5} \tau^{2}\left(1-\frac{912}{2625} \alpha \beta\right)-\frac{27}{25}\left(1-\frac{432}{675} \alpha \beta\right)\right] \\
& P_{1}(\omega, k)=|k|^{8} \tau^{2}\left[\frac{13}{3} \tau^{6}-\frac{1094}{75} \tau^{4}+\frac{1381}{125} \tau^{2}\left(1-\frac{2032}{6905} \alpha \beta\right)-\frac{264}{125}\left(1-\frac{143}{330} \alpha \beta\right)\right], \\
& P_{2}(\omega, k)=|k|^{7} \tau\left[\frac{67}{9} \tau^{6}-\frac{497}{25} \tau^{4}+\frac{3943}{375} \tau^{2}\left(1-\frac{832}{3943} \alpha \beta\right)-\frac{159}{125}\left(1-\frac{48}{159} \alpha \beta\right)\right] \\
& P_{3}(\omega, k)=|k|^{6}\left[\frac{19}{3} \tau^{6}-\frac{2908}{225} \tau^{4}+\frac{13}{3} \tau^{2}\left(1-\frac{32}{325} \alpha \beta\right)-\frac{6}{25}\right] \\
& P_{4}(\omega, k)=|k|^{5} \tau\left[\frac{8}{3} \tau^{4}-\frac{178}{45} \tau^{2}+\frac{2}{3}\right], \quad P_{5}(\omega, k)=\frac{4}{9}|k|^{4} \tau^{2}\left(\tau^{2}-1\right),
\end{aligned}
$$

где $\omega=|k| \tau, \alpha=k_{1}^{2}, \beta=k_{2}^{2}$. Нетрудно проверить, что для любых $0 \leqslant \alpha, \beta \leqslant 1$, $\alpha+\beta=1$, гиперболический пучок длины $N=5$, определяемый цепочкой $(11)$, является нестрого гиперболическим.

2. Задача Коши. Основная идея введения неравновесных переменных связана с тем, что для теоретического описания [1]-[3] процессов со строго градиентным и быстрьм изменением параметров необходимо большее число переменных. Базовьми величинами являются: плотность $\varrho$, скорость $v$ и температура $T$, отвечающие системе уравнений Эйлера газовой динамики, как невозмущенной (предельной) системы. Однородная часть системы (1)-(8) является гиперболической и правая часть системы вектор $P$ является линейной вектор-функцией относительно неравновесных величин. Первые пять компонент вектора $P$ тождественно равны нулю, что соответствует пяти законам сохранения - массы, момента и энергии. В равновесном состоянии все компоненты вектора правой части равны нулю и, следовательно, формально все неравновесные величины 
равны нулю. Не формально это утверждение требует исследования предельного перехода по параметру $B \rightarrow \infty$, т.е. исследования условий типа Лакса глобальной устойчивости решений задачи Коши или смешанной задачи.

В статье [6] было доказано, что в одномерном случае для дисперсионного полинома 13-моментной системы Эрмита-Града справедлив аналог классической теоремы Эрмита-Биллера [7] об устойчивых полиномах. Более того, условия, которым удовлетворяет этот дисперсионный полином, являются необходимьми и достаточными условиями устойчивости строго гиперболической тройки полиномов. В 13-моментном случае дисперсионное уравнение

$$
P^{(13)}(\omega, k)=P_{0}(\omega, k)-i \gamma_{1} B P_{1}(\omega, k)-\gamma_{2} B^{2} P_{2}(\omega, k)=0
$$

где $\gamma_{1}=4 / 3, \gamma_{2}=32 / 75, \tau=\omega+\mu_{E} k$ и $\mu_{E}=v_{E} / c_{s}$ - число Маха, определяет полиномиальный пучок тройки строго гиперболических полиномов

$$
P_{0}=\tau\left(\tau^{4}-\frac{78}{25} \tau^{2} k^{2}+\frac{27}{25} k^{4}\right), \quad P_{1}=\tau^{4}-\frac{33}{25} \tau^{2} k^{2}+\frac{6}{25} k^{4}, \quad P_{2}=\tau\left(\tau^{2}-k^{2}\right)
$$

для которого справедлив следующий результат [6].

ПРЕДЛОЖЕНИЕ 2.1. Пусть полиномиальный пучок (12) определяет строго гиперболическую тройку однородных полиномов, стариие коэффициенты которых $P_{5}(1,0)=P_{4}(1,0)=P_{3}(1,0)=1$ и постоянные $\gamma_{1}>0, \gamma_{2}>0$. Тогда для любого вещественного $k \in \mathbb{R}^{1}, k \neq 0$, корни $\omega(k)$ дисперсионного уравнения (12) лежсат в верхней полуплоскости $\operatorname{Im} \omega>0$ комплексной плоскости.

Корни соседних полиномов $P_{j}$ в 13-моментном случае $\left(\omega_{1}^{ \pm}=k\left(\mu_{E} \pm 1\right), \omega_{2}=k \mu_{E}\right.$ для полинома $P_{2}$; для полинома $P_{1}$ имеем $\omega_{1}^{ \pm}=k\left(\mu_{E} \pm \tilde{b}_{1}\right), \omega_{2}^{ \pm}=k\left(\mu_{E} \pm \tilde{b}_{2}\right)$; для $P_{0}$ имеем $\left.\omega_{1}^{ \pm}=k\left(\mu_{E} \pm \tilde{c}_{1}\right), \omega_{2}^{ \pm}=k\left(\mu_{E} \pm \tilde{c}_{2}\right), \omega_{3}=k \mu_{E}\right)$ взаимно разделяют друг друга. Здесь

$$
\tilde{c}_{1}=1.6503, \quad \tilde{c}_{2}=0.6297, \quad \tilde{b}_{1}=1.1, \quad \tilde{b}_{2}=0.22,
$$

где $\mu_{E}<b_{2}<c_{2}<b_{1}<c_{1}$ и $\mu_{E}<b_{2}<1<b_{1}$. Таким образом, в линейном приближении 13-моментная система является глобально устойчивой. В 20-моментном случае имеем строго гиперболический пучок

$$
\begin{gathered}
P^{(20)}(\omega, k)=P_{0}-i \gamma_{1} B P_{1}-\gamma_{2} B^{2} P_{2}+i \gamma_{3} B^{3} P_{3}=0 \\
P_{0}(\omega, k)=\tau^{6}-\frac{21}{5} \tau^{4} k^{2}+\frac{81}{25} \tau^{2} k^{4}-\frac{81}{125} k^{6}, \quad P_{1}(\omega, k)=\left(\tau^{4}-\frac{258}{95} \tau^{2} k^{2}+\frac{441}{445} k^{4}\right) \\
P_{2}(\omega, k)=\tau^{4}-\frac{164}{95} \tau^{2} k^{2}+\frac{27}{95} k^{4}
\end{gathered}
$$

$P_{3}$ то же, что $P_{2}$ в 13 -моментном случае, $\gamma_{1}=19 / 6, \gamma_{2}=19 / 6, \gamma_{3}=1$. Справедливо следующее обобщение этой теоремы на 20-моментньй случай. 
ПРЕДЛОЖЕНИЕ 2.2. Пусть полиномиальный пучок (15) является строго гиперболическим пучком длины $N=3$. Старшие коэффичиенты полиномов пучка $P_{6}(1,0)=P_{5}(1,0)=P_{4}(1,0)=P_{3}(1,0)=1$, постоянные в $(15) \quad \gamma_{j}>0 u$ $\gamma_{1} \gamma_{2}-\gamma_{3}>0$. Тогда для любого вещественного $k \in \mathbb{R}^{1}, k \neq 0$, корни $\omega(k)$ дисперсионного уравнения (15) лехат в верхней полуплоскости $\operatorname{Im} \omega>0$ комплексной плоскости.

Следствием этих результатов является иерарахия волн [8] (иерархия медленных корней и корней погранслойного типа) для решения задачи Коши системы (1)-(8).

3. Смешанная задача. Другой круг задач моментной теории связан со специфическими трудностями смешанных задач для систем моментов. Часть зависимых переменных (моменты высших порядков) не имеют интуитивного физического смысла. Такие переменные не могут быть определены из эксперимента, что должно учитьваться в структуре краевых условий. Для формулировки смешанной задачи требуются подходящие (корректные с физической точки зрения) граничные условия. Так, например, для разряженного газа возможен скачок температуры на стенке и возможно появление скорости проскальзьвания. Впервые граничные условия, описьвающие эти феномены, были предложены в [3]. Исследуем линеаризацию этих граничных условий в окрестности состояния равновесия. Для простоты рассмотрим эту смешанную задачу в 1-D случае в четверти плоскости $\mathbb{R}^{++}=\{(x, t): t>0, x>0\}$. Тогда в безразмерных переменных для 13- и 20-моментных систем получим следующие условия:

$$
\begin{aligned}
& \left.\left(\alpha^{\theta} q_{1}+\beta^{p} p_{\langle 11\rangle}\right)\right|_{x=0}=T_{W}-\left.T\right|_{x=0}, \\
& \left.\left(\alpha^{\theta} q_{1}+\beta^{p} p_{\langle 11\rangle}+\beta^{\varrho} \varrho_{\langle 111\rangle}\right)\right|_{x=0}=T_{W}-\left.T\right|_{x=0} \text {. }
\end{aligned}
$$

Здесь

$$
\alpha^{\theta}=\frac{1}{2} \sqrt{\frac{5 \pi}{6}}, \quad \beta^{p}=\frac{1}{15}, \quad \beta^{\varrho}=\frac{3}{10} .
$$

Потребуем также, чтобы $\left.v_{1}\right|_{x=0}=v_{b}(t),\left.\varrho\right|_{x=0}=\varrho_{b}(t),\left.p_{\langle 11\rangle}\right|_{x=0}=0$ в 13-моментном случае и дополнительно $\left.\varrho_{\langle 111\rangle}\right|_{x=0}=0$ в 20 -моментном случае. Как мы видим, линеаризованные условия, введенные в [9], являются частным случаем условий вида

$$
\left.\mathcal{B U}\right|_{x=0}=\mathcal{F}_{b}
$$

Здесь $\mathcal{F}_{b}(t)$ граничные данные задачи, $\mathcal{B}$ является $3 \times\left(3+n_{g}\right)$ матрищей с постоянньми коэффициентами, $n_{g}=2$ и $n_{g}=3$ в 13- и 20-моментном случаях соответственно.

КОМмЕНТАРИЙ 1. Ниже мы получим условие корректности этого граничного условия и как следствие - условие корректности граничного условия, предложенного в [3]. Пусть $N_{c}^{+}$- число положительных постоянных $\mu_{E}+\tilde{c}_{j}>0$ и $N_{a}^{+}$- число положительных постоянных $\mu_{E}+\tilde{a}_{j}>0$. Мы покажем, что число $N^{+}$необходимых условий корректной задачи $(18)$ равно $N_{c}^{+}$- числу входящих в область $\mathbb{R}^{++}$характеристик старшей части линеаризованной системы. Точнее $N^{+}=N_{a}^{+}+N_{b}^{+}$. Здесь $N_{a}^{+}$- число входящих в область $\mathbb{R}^{++}$характеристик линеаризованной системы уравнений Эйлера, $N_{b}^{+}$равно числу линейно независимых устойчивых решений типа погранслоя, локализованных в $1 / B$ окрестности гранищы. 
Рассмотрев нулевые начальные данные, продолжим решение и краевые данные нулем для $t<0$, и сведем преобразованием Фурье смешанную задачу в четверти плоскости $\mathbb{R}^{++}$к граничной задаче для системы линейных обыкновенных дифференциальных уравнений на полупрямой $\mathbb{R}^{+}=\{x>0\}$, общее решение которой представляется в виде

$$
U(x, t)=\frac{1}{2 i \pi} \int_{\mathcal{B}} \sum_{j=1}^{N^{+}} G_{j}(B \xi) R_{j}(B \xi) e^{i B\left(\xi t+K_{j}(\xi) x\right)} d \xi, \quad t>0
$$

где контур интегрирования $\mathcal{B}$ находится вьше всех особенностей подьнтегрального выражения в комплексной $\xi$-плоскости. Здесь $K_{j}, j=1, \ldots, 3+n_{g}$, определяются корнями $k(\omega, B)=B K(\xi), \omega=B \xi$ относительно $k=k(\omega)$ дисперсионного уравнения смешанной задачи в 13 - и 20-моментном случаях соответственно, $\mathcal{R}_{j}$ - столбцы при $x=t=0$ фундаментальной матрицы $\mathcal{F}(t, x, \xi)$ порядка $N^{+} \times\left(3+N_{g}\right)$ устойчивых плоских решений $R_{j}(B \xi) e^{i B\left(\xi t+B K_{j}(\xi) x\right)}, \operatorname{Im} K_{j}>0$, полученной системы обыкновенных дифференциальных уравнений. Неизвестные функции $G_{j}$ однозначно определяются из граничного условия $(16),(17)$ как решение системы $\mathcal{B R}(\xi) \mathcal{G}(\xi)=\widetilde{\mathcal{F}}_{b}(\xi)$, если вьполнен аналог условия Шапиро-Лопатинского [10]

$$
\operatorname{det}(\mathcal{B R}) \neq 0, \quad \operatorname{Im} \xi \geqslant c_{0},
$$

для некоторого $c_{0} \geqslant 0$. Здесь $\widetilde{\mathcal{F}}_{b}(\xi)$ - преобразование Фурье граничных данных. Тем самым, для смешанной задачи мы получим аналог теоремы Вишика-Люстерника [11] о малых сингулярных возмущениях обших эллиптических задач, дав определение условия Шапиро-Лопатинского о корректности смешанной задачи. Условие (20) мы исследуем ниже. Перейдем к доказательству утверждения о независимости числа устойчивых корней $K_{j}(\xi)$ от вещественного параметра $\xi \in \mathbb{R}^{1}$.

4. Полиномиальный пучок смешанной задачи. Теперь исследуем полиномиальньй пучок, определяемьй смешанной задачей в 13-моментном и в 20-моментном случаях. В 13-моментном случае дисперсионное уравнение смешанной задачи имеет вид

$$
\begin{gathered}
P_{0}(K(\xi), \xi)-P_{2}(K(\xi), \xi)-i P_{1}(K(\xi), \xi)=0 \\
P_{0}=\prod_{j=1}^{5}\left(\xi+\left(\mu_{E}+\tilde{c}_{j}\right) K(\xi)\right), \quad P_{1}=\prod_{j=1}^{4}\left(\xi+\left(\mu_{E}+\tilde{b}_{j}\right) K(\xi)\right) \\
P_{2}=\prod_{j=1}^{3}\left(\xi+\left(\mu_{E}+\tilde{a}_{j}\right) K(\xi)\right) .
\end{gathered}
$$

Переход от дисперсионного уравнения задачи Коши к дисперсионному уравнению смешанной задачи в 20-моментном случае

$$
P_{0}(K(\xi), \xi)-P_{2}(K(\xi), \xi)-i\left(P_{1}(K(\xi), \xi)-P_{3}(K(\xi), \xi)\right)=0
$$

тот же, что и в 13-моментном. Как мы покажем ниже, свойства корней дисперсионных уравнений $(21),(22)$ определяют поведение решений смешанной задачи. 
ТЕОремА 4.1. Пусть полиномиальный пучок (14) (соответственно (15)) удовлетворяет условиям предложсения 2.1 (предложсения 2.2). Дополнительно в 13моментном случае потребуем, чтобы

$$
\mu_{E} \neq 0, \quad\left(\mu_{E} \pm 1\right) \neq 0, \quad\left(\mu_{E} \pm \tilde{c}_{j}\right) \neq 0, \quad\left(\mu_{E} \pm \tilde{b}_{j}\right) \neq 0, \quad j=1,2
$$

а в 20-моментном случае

$$
\begin{gathered}
\mu_{E} \neq 0, \quad\left(\mu_{E} \pm 1\right) \neq 0 \\
\left(\mu_{E} \pm \tilde{c}_{j}\right) \neq 0, \quad\left(\mu_{E} \pm \tilde{b}_{j}\right) \neq 0, \quad\left(\mu_{E} \pm \tilde{d}_{j}\right) \neq 0, \quad j=1,2 \\
\gamma_{1} \gamma_{2} \prod_{j=1,2}\left|\mu_{E}\right|\left|\left(\mu_{E} \pm \tilde{b}_{j}\right)\right|\left|\left(\mu_{E} \pm \tilde{d}_{j}\right)\right|-\gamma_{3}\left|\mu_{E}\right| \prod_{k=1,2}\left|\left(\mu_{E} \pm \tilde{c}_{j}\right)\right|\left|\left(\mu_{E} \pm 1\right)\right|>0
\end{gathered}
$$

Тогда в 13- и 20-моментных случаях для любого $\xi \in \mathbb{R}^{1}, \xi \neq 0$, число $N^{ \pm}(\xi)$ корней дисперсионного уравнения с полохительной и отрицательной мнимыми частями не зависит от $\xi \in \mathbb{R}^{1}, \xi \neq 0$, и равно $N_{c}^{ \pm}=N_{a}^{ \pm}+N_{b}^{ \pm}$.

Как мы отмечали вьше, $N_{c}^{ \pm}, N_{a}^{ \pm}$равны числу положительных (отрицательных) значений $\mu_{E},\left(\mu_{E} \pm \tilde{c}_{j}\right), j=1,2$, и $\mu_{E},\left(\mu_{E} \pm 1\right)$ соответственно; $N_{b}^{ \pm}$- число погранслойных корней с положительными (отрицательными) мнимыми частями.

Докажем теорему 4.1. Для этого покажем, что в 13- и 20-моментных случаях для любого вещественного $\xi>0$ корни дисперсионного уравнения лежат в верхней и нижней полуплоскостях комплексной плоскости, т.е. $N^{+}$корней удовлетворяют условию $\operatorname{Im} K(\xi)>0$ и $3+N_{g}-N^{+}$корней таковы, что $\operatorname{Im} K(\xi)<0$. Случай $\xi<0$ исследуется аналогично. Начнем с 13-моментного случая. Справедлива следующая лемма.

ЛЕмма 4.1. Пусть полиномиальный пучок (21) удовлетворяет условиям теоремы 4.1. Тогда пять корней (21) имеют следующую структуру: для пяти корней $K_{j}(\xi)$ в высокочастотном приближении при $|\xi| \geqslant \delta_{1}$ справедливо следующее разложение:

$$
\begin{gathered}
K_{h, j}=-\frac{\xi}{c_{j}}+i \kappa_{j}^{h}+O\left(\frac{1}{|\xi|}\right), \quad j=1, \ldots, 5, \\
c_{1}=\mu_{E}+\tilde{c}_{1}, \quad c_{2}=\mu_{E}+\tilde{c}_{2}, \quad c_{3}=\mu_{E}, \quad c_{4}=\mu_{E}-\tilde{c}_{2}, \quad c_{5}=\mu_{E}-\tilde{c}_{1}, \\
\kappa_{j}^{h}=\frac{\gamma_{1} \prod_{k=1}^{4}\left(b_{k}-c_{j}\right)}{c_{j} \prod_{k \neq j}\left(c_{k}-c_{j}\right)} \\
b_{1}=\mu_{E}+\tilde{b}_{1}, \quad b_{2}=\mu_{E}+\tilde{b}_{2}, \quad b_{3}=\mu_{E}-\tilde{b}_{2}, \quad b_{4}=\mu_{E}-\tilde{b}_{1}, \\
a_{1}=\mu_{E}+1, \quad a_{2}=\mu_{E}, \quad a_{3}=\mu_{E}-1 .
\end{gathered}
$$

В низкочастотном приближении при $|\xi| \leqslant \delta_{2}$ для трех корней имеем разложения вида

$$
K_{l, j}=-\frac{\xi}{a_{j}}+i \kappa_{j}^{l} \xi^{2}+O\left(|\xi|^{3}\right), \quad \kappa_{j}^{l}=\frac{\gamma_{1} \prod_{k=1}^{4}\left(b_{k}-a_{j}\right)}{a_{j}^{3} \gamma_{2} \prod_{k \neq j}\left(a_{k}-a_{j}\right)}, \quad j=1,2,3
$$


Для двух $K_{j}(\xi), j=4,5$, так называемых погранслойных корней, в низкочастотном приближсении справедливы разложения $K_{j}=\kappa_{b, j}^{l}+O(|\xi|), j=4,5$. Здесь $\kappa_{b, j}^{l}$, $j=4,5$, являются корнями уравнения

$$
\left(\kappa_{b}^{l}\right)^{2}-i \alpha_{1} \kappa_{b}^{l}-\alpha_{2}=0, \quad \alpha_{1}=\gamma_{1} \frac{\prod_{j=1}^{4} b_{j}}{\prod_{j=1}^{5} c_{j}}, \quad \alpha_{2}=\gamma_{2} \frac{\prod_{j=1}^{3} a_{j}}{\prod_{j=1}^{5} c_{j}} .
$$

Построение асимптотических разложений проводится прямым вычислением регулярной асимптотики. Из условия взаимного разделения корней строго гиперболической тройки полиномов следует, что $c_{j} \kappa_{j}^{h}>0, j=1, \ldots, 5, a_{j} \kappa_{j}^{l}>0, j=1,2,3$. Таким образом, для больших $|\xi| \gg 1$ в верхней полуплоскости находится $N_{c}^{+}$(соответственно в нижней $N_{c}^{-}$) корней. Также для малых $|\xi| \ll 1$ в верхней полуплоскости находится $N_{a}^{+}$ (соответственно в нижней $N_{a}^{-}$) корней. Теперь для $|\xi| \ll 1$ рассмотрим погранслойные корни, главные части которых являются решениями уравнения (29). После замены $\kappa_{b}^{l}=-i z$ это уравнение может иметь различную структуру в зависимости от чисел $N_{c}^{-}, N_{b}^{-}, N_{a}^{-}$отрицательных значений $c_{j}, b_{j}, a_{j}$. Возможны следующие комбинации:

\begin{tabular}{c|c|c|c|c|c|c|}
\hline$N_{c}^{+}$ & $N_{b}^{+}$ & $N_{a}^{+}$ & $\operatorname{sign} \alpha_{1}$ & $\operatorname{sign} \alpha_{2}$ & $N_{b}^{+}$ & $P$ \\
\hline 0 & 0 & 0 & + & + & 2 & $P_{1}(z)$ \\
1 & 0 & 0 & - & - & 1 & $P_{2}(z)$ \\
1 & 1 & 0 & + & - & 1 & $P_{3}(z)$ \\
1 & 1 & 1 & + & + & 2 & $P_{1}(z)$ \\
2 & 1 & 1 & - & - & 1 & $P_{2}(z)$ \\
2 & 2 & 1 & + & - & 1 & $P_{3}(z)$ \\
3 & 2 & 2 & - & - & 1 & $P_{2}(z)$ \\
3 & 3 & 2 & + & - & 1 & $P_{3}(-z)$ \\
4 & 3 & 2 & - & + & 0 & $P_{4}(-z)$ \\
4 & 3 & 3 & - & - & 1 & $P_{2}(-z)$ \\
4 & 4 & 3 & + & - & 1 & $P_{3}(-z)$ \\
5 & 4 & 3 & - & + & 0 & $P_{4}(-z)$ \\
$P_{1}(z)=z^{2}+\left|\alpha_{1}\right| z+\left|\alpha_{2}\right|$, & $P_{2}(z)=z^{2}-\left|\alpha_{1}\right| z-\left|\alpha_{2}\right|$,
\end{tabular}
$P_{3}(z)=z^{2}+\left|\alpha_{1}\right| z-\left|\alpha_{2}\right|, \quad P_{4}(z)=z^{2}-\left|\alpha_{1}\right| z+\left|\alpha_{3}\right|$,

$N_{b}^{+}$- число корней полинома $P_{j}$ с отрищательной вещественной частью.

Отсюда следует утверждение: для любого достаточно малого $|\xi| \ll 1$ число корней в верхней полуплоскости (соответственно в нижней полуплоскости) равно $N_{c}^{+}=N_{a}^{+}+N_{b}^{+}$ и $N_{c}^{-}=N_{a}^{-}+N_{b}^{-}$. Таким образом, для любого вещественного $\xi>0$ вне отрезка $\delta_{2} \leqslant \xi \leqslant \delta_{1}$ нет вещественных и чисто мнимьх корней $(\operatorname{Im} K(\xi)=0)$ дисперсионного уравнения и их число в верхней и нижней полуплоскости не зависит от $\xi$. Несуществование вешественных корней в 13-моментном случае доказывается просто. Если существует вещественньй корень, то $\left(P_{0}-P_{2}\right)\left(\xi^{*}, K\left(\xi^{*}\right)\right)=P_{1}\left(\xi^{*}, K\left(\xi^{*}\right)\right)=0, \xi^{*} \neq 0$, для некоторого $k \in\{1, \ldots, 4\}$, где $K\left(\xi^{*}\right)=-\xi^{*} / b_{k}$. Отсюда получаем

$$
\left(P_{0}-P_{2}\right)\left(\xi^{*}, K\left(\xi^{*}\right)\right)=\frac{\left(\xi^{*}\right)^{3}}{b_{k}^{3}}\left(\frac{\left(\xi^{*}\right)^{2}}{b_{k}^{2}} \prod_{j=1}^{5}\left(b_{k}-c_{j}\right)-\prod_{j=1}^{3}\left(b_{k}-a_{j}\right)\right) \neq 0
$$


так как в силу условия взаимного разделения корней имеем

$$
\frac{\left(\xi^{*}\right)^{2}}{b_{k}^{2}} \prod_{j=1}^{5}\left(b_{k}-c_{j}\right)-\prod_{j=1}^{3}\left(b_{k}-a_{j}\right)>0
$$

что противоречит уравнению $\left(P_{0}-P_{2}\right)\left(\xi^{*}, K\left(\xi^{*}\right)\right)=0$. Следовательно, для $\xi>0$ траектории корней не пересекают вешественной оси. Отсюда следует, что для любого $\xi>0$ в верхней полуплоскости находится $N_{c}^{+}$корней и соответственно $N_{c}^{-}$корней находится в нижней полуплоскости.

Таким образом, для любого $\xi \neq 0$ траектории корней остаются в верхней или нижней полуплоскостях комплексной плоскости. Более того, для больших значений $\xi \gg 1$ траектории корней $K_{j}(\xi)$ в верхней полуплоскости комплексной плоскости находятся одна над другой по возрастанию номера $j=1, \ldots, 5$, т.е. $\operatorname{Im} K_{j+1}>\operatorname{Im} K_{j}, j=1, \ldots, 4$, и движутся к мнимой оси с убывающими (по возрастанию номера $j$ ) скоростями проекций на вешественную ось, оставаясь в верхней полуплоскости комплексной плоскости. Предложение 4.1 в 13-моментном случае доказано.

КОмМЕНТАРИЙ 2. На самом деле, можно ввести понятие непрерывной ветви корня. Первой ветвью (первым корнем $K_{1}$ ) назовем огибающую снизу всех корней. Затем рассмотрим замькание области вьше этой огибающей и назовем второй ветвью (вторым корнем $K_{2}$ ) огибающую снизу всех корней в этом множестве и т.д. Тогда ветви (корни) (21) входят в окрестность малых значений $\xi>0, \xi \ll 1$, по кривым, прилегающим в главном (до порядка $\xi^{3}$ ) к параболам

$$
\operatorname{Im} K_{j}=\kappa_{j}^{l}\left(\operatorname{Re} K_{j}\right)^{2}, \quad \kappa_{3}^{l}>\kappa_{2}^{l}>\kappa_{1}^{l}>0, \quad \kappa_{j}^{l}=-\frac{1}{a_{j}^{2}} \frac{\prod_{j=1}^{k}\left(b_{k}-a_{j}\right)}{\prod_{k \neq j}\left(a_{k}-a_{j}\right)}
$$

При $\xi=0$ имеем трехкратньй корень $K_{j}(0)=0, j=1,2,3$, и два простых погранслойных корня $K_{j}(0)=\kappa_{b, j}^{i}, j=4,5$. Возникает проблема описания корней в промежуточных состояниях, когда $\delta_{2} \leqslant|\xi| \ll \delta_{1}$, что чрезвычайно важно в решении задачи о иерархии волн [12] в системах моментов неравновесной термодинамики на больших временах и при $B \rightarrow \infty$. Гипотеза состоит в том, что в промежуточных состояниях вещественные части корней последовательно находятся в окрестностях корней промежуточных гиперболических операторов цепочки, определяемой дисперсионным уравнением. Насколько это верно, покажут дальнейшие исследования.

В 20-моментном случае так же, как вьше, получим следуюоее утверждение.

ЛЕмма 4.2. Пусть полиномиальный пучок (22) удовлетворяет условиям теорем̈ $4.2 u$

$$
\begin{gathered}
\mu_{E} \neq 0, \quad\left(\mu_{E} \pm 1\right) \neq 0, \\
\left(\mu_{E} \pm \tilde{c}_{j}\right) \neq 0, \quad\left(\mu_{E} \pm \tilde{b}_{j}\right) \neq 0, \quad\left(\mu_{E} \pm \tilde{d}_{j}\right) \neq 0, \quad j=1,2, \\
\gamma_{1} \gamma_{2} \prod_{j=1,2}\left|\mu_{E}\right|\left|\left(\mu_{E} \pm \tilde{b}_{j}\right)\right|\left|\left(\mu_{E} \pm \tilde{d}_{j}\right)\right|-\gamma_{3}\left|\mu_{E}\right| \prod_{k=1,2}\left|\left(\mu_{E} \pm \tilde{c}_{j}\right)\right|\left|\left(\mu_{E} \pm 1\right)\right|>0 .
\end{gathered}
$$


Тогда шесть $K_{j}(\xi)$ корней уравнения (22) имеют высокочастотное и низкочастотное асимптотические приближения следующего вида: для четырех корней $K_{j}(\xi)$ в высокочастотном приближсении $|\xi| \gg 1$ справедливо следующее разложенuе:

$$
K_{j}(\xi)=-\frac{\xi}{c_{j}}+i \frac{\gamma_{1} \prod_{k=1}^{5}\left(b_{k}-c_{j}\right)}{c_{j} \prod_{k \neq j}\left(c_{k}-c_{j}\right)}+O\left(\frac{1}{|\xi|}\right), \quad j=1, \ldots, 6 .
$$

В низкочастотном приближсении $|\xi| \ll 1$ имеем

$$
K_{j}(\xi)=-\frac{\xi}{a_{j}}+i \frac{\gamma_{2} \prod_{k=1}^{4}\left(d_{k}-a_{j}\right)}{a_{j}^{3} \gamma_{3} \prod_{k \neq j}\left(a_{k}-a_{j}\right)} \xi^{2}+O\left(|\xi|^{3}\right), \quad j=1,2,3
$$

В этом приблиэении есть еще три погранслойных корня с асимптотическими разложсениями вида $K_{j}(\xi)=\kappa_{b, j}^{l}+O(|\xi|), j=4,5,6$, главные части которых определяются решениями кубического уравнения

$$
\prod_{j=1}^{6} c_{j}\left(\kappa_{b}^{l}\right)^{3}-i \gamma_{1} \prod_{j=1}^{5} b_{j}\left(\kappa_{b}^{l}\right)^{2}-\gamma_{2} \prod_{j=1}^{4} d_{j} \kappa_{b}^{l}+i \gamma_{3} \prod_{j=1}^{2} a_{j}=0
$$

Доказательство этой леммы проводится прямым вычислением регулярных асимптотик. Теперь исследуем уравнение для погранслойных корней (34). Положим $\kappa_{b}^{l}=-i z$ и приведем классификацию структуры этого уравнения в зависимости от чисел $N_{c}^{-}, N_{b}^{-}$, $N_{d}^{-}, N_{a}^{-}$- количества отрицательных значений $c_{j}, b_{j}, d_{j}, a_{j}$ :

\begin{tabular}{|c|c|c|c|c|c|c|c|c|}
\hline$N_{c}^{-}$ & $N_{b}^{-}$ & $N_{d}^{-}$ & $N_{a}^{-}$ & $\operatorname{sign} \alpha_{1}$ & $\operatorname{sign} \alpha_{2}$ & $\operatorname{sign} \alpha_{3}$ & $N_{b}^{+}$ & $P$ \\
\hline 0 & 0 & 0 & 0 & + & + & + & 3 & $P_{1}(z)$ \\
1 & 0 & 0 & 0 & - & - & - & 2 & $P_{2}(z)$ \\
1 & 1 & 0 & 0 & + & - & - & 2 & $P_{3}(z)$ \\
1 & 1 & 1 & 0 & + & + & - & 2 & $P_{4}(z)$ \\
1 & 1 & 1 & 1 & + & + & + & 3 & $P_{1}(z)$ \\
2 & 1 & 1 & 1 & - & - & - & 2 & $P_{2}(z)$ \\
2 & 2 & 1 & 1 & + & - & - & 2 & $P_{3}(z)$ \\
2 & 2 & 2 & 1 & + & + & - & 2 & $P_{4}(z)$ \\
4 & 3 & 2 & 2 & - & + & + & 1 & $P_{4}(-z)$ \\
4 & 3 & 3 & 2 & - & - & + & 1 & $P_{3}(-z)$ \\
4 & 4 & 3 & 2 & + & - & + & 1 & $P_{2}(-z)$ \\
5 & 4 & 3 & 2 & - & + & - & 0 & $P_{1}(-z)$ \\
5 & 4 & 3 & 3 & - & + & + & 1 & $P_{4}(-z)$ \\
5 & 4 & 4 & 3 & + & - & + & 1 & $P_{3}(-z)$ \\
5 & 5 & 4 & 3 & + & - & + & 1 & $P_{2}(-z)$ \\
6 & 5 & 4 & 3 & - & + & - & 0 & $P_{1}(-z)$ \\
\hline
\end{tabular}




$$
\begin{array}{ll}
P_{1}(z)=z^{3}+\left|\alpha_{1}\right| z^{2}+\left|\alpha_{2}\right| z+\left|\alpha_{3}\right|, & P_{2}(z)=z^{3}-\left|\alpha_{1}\right| z^{2}-\left|\alpha_{2}\right| z-\left|\alpha_{3}\right| \\
P_{3}(z)=z^{3}+\left|\alpha_{1}\right| z^{2}-\left|\alpha_{2}\right| z-\left|\alpha_{3}\right|, & P_{4}(z)=z^{3}+\left|\alpha_{1}\right| z^{2}+\left|\alpha_{2}\right| z-\left|\alpha_{3}\right|
\end{array}
$$

$N_{b}^{+}$- число корней полинома $P_{j}$ с отрищательной вещественной частью.

ЛЕмма 4.3. Пусть $\left|\alpha_{1} \alpha_{2}\right|-\left|\alpha_{3}\right|>0$. Тогда все корни полинома $P_{1}$ имеют отрицательные вещ,ственные части, полиномы $P_{j}, j=2,3,4$, имеют один положительный корень и два корня с отрицательными вещественными частями.

Утверждение о свойствах полинома $P_{1}$ является следствием правила Раусса-Гурвица. Для полинома $P_{2}$ имеем $P_{3}(0)=-\left|\alpha_{3}\right|<0, P_{3}\left(-\left|\alpha_{1}\right|\right)=\left|\alpha_{1} \alpha_{2}\right|-\left|\alpha_{3}\right|>0$, откуда следует существование одного положительного корня и двух отрицательных. Для полиномов $P_{2}, P_{4}$ из условия $P_{j}(0)=-\left|\alpha_{3}\right|<0$ также следует существование положительного корня $\kappa>0$. Тогда эти полиномы можно представить в виде

$$
\begin{gathered}
P_{4}(z)=(z-\kappa)\left(y^{2}+\left(3 \kappa+\left|\alpha_{1}\right|\right) y+P_{j}^{\prime}(\kappa)\right), \quad y=z-\kappa, \\
P_{2}(z)=(z-\kappa)\left(y^{2}+\left(3 \kappa-\left|\alpha_{1}\right|\right) y+P_{j}^{\prime}(\kappa)\right),
\end{gathered}
$$

где $P_{j}^{\prime}(\kappa)>0, j=1,2$. Так как $3 \kappa+\left|\alpha_{1}\right|>0$, в первом случае вещественные части двух других корней отрицательны. Во втором случае имеем $P_{2}\left(\left|\alpha_{1}\right|\right)=-\left|\alpha_{2} \alpha_{1}\right|-\left|\alpha_{3}\right|<0$, т.е. $\kappa>\left|\alpha_{1}\right|$; следовательно, $3 \kappa-\left|\alpha_{1}\right|>0$, откуда снова получаем, что вешественные части двух других корней полинома $P_{2}$ отрицательны. Лемма доказана.

Таким образом, так же, как в 13-моментном случае, для значений $|\xi| \geqslant \delta_{1}^{-1},|\xi| \leqslant \delta_{1}$, $\delta_{1}$ достаточно мало, $N^{+}$корней уравнения $(22)$ для $n_{1}>0$ лежат в верхней и соответственно $N^{-}=6-N^{+}$корней в нижней полуплоскостях комплексной плоскости (тоже справедливо для $\left.n_{1}<0\right)$. Число их не зависит от $n_{1}$ и равно $N_{c}^{+}$и $N_{c}^{-}$соответственно. Завершение доказательства этого предложения проводится аналогично 13-моментному случаю. Существенно сложнее доказательство отсутствия вещественных корней (22), которое мы приведем в отдельной статье. Как мы отмечали вьше, эти результаты позволяют, используя стандартную процедуру построения общего решения линеаризованной смешанной задачи, построить аналог условия Шапиро-Лопатинского для эллиптических краевых задач [10].

5. Условие Шапиро-Лопатинского. Исследуем наиболее интересньй случай линеаризации одномерной 13-моментной системы при $N_{c}^{+}=4>N_{a}^{+}=2$, т.е. случай, когда требуется на два граничных условия больше, чем для предельной линеаризованной системы уравнений Эйлера. Пусть $\mathcal{R}^{v}, \mathcal{R}^{\varrho}, \mathcal{R}^{U}, \mathcal{R}^{p_{\langle 11\rangle}}, \mathcal{R}^{q_{1}}-$ строки фундаментальной матрищы $\mathcal{F}$ устойчивых решений при $t=x=0$, определяемые координатами величин $v, \varrho, U, p_{\langle 11\rangle}, q_{1}$ соответственно. В 13 -моментном случае, когда $N_{c}^{+}=4$, рассмотрим матрицу $\mathcal{R}^{D}$, строками которой являются $\mathcal{R}^{v}, \mathcal{R}^{\varrho}, \mathcal{R}^{U}, \mathcal{R}^{p}\langle 11\rangle$, и матрицу $\mathcal{R}^{\theta}$, строки которой $\mathcal{R}^{v}, \mathcal{R}^{\varrho}, \mathcal{R}^{U}, \mathcal{R}^{q_{1}}$.

ЛЕмма 5.1. В 1-D 13-моментном случае $\left(N^{+}=4\right)$ для почти всех $\xi \in \mathbb{R}^{1}$, $\xi \neq 0$, исключая случай кратности устойчивых корней, $\operatorname{det} \mathcal{R}^{\theta}(\xi) \neq 0$.

Как следствие этой леммы можно сформулировать следующее утверждение. 
ТЕОРемА 5.1. Пусть выполнены условия предложения 4.1 (о числе устойчивых корней 13-моментного пучка). Тогда для достаточно большого $\alpha^{\theta}$, т.е. достаточно малого коэффичиента согласования $\theta \ll 1$, краевая задача (17) корректна (справедливо условие Шапиро-Лопатинского (20)).

ОПРЕДЕЛЕНИЕ 5.1. Корректную смешанную задачу назовем равновесной, если в нормах корректности задачи неравновесные переменные стремятся к нулю при $B \rightarrow \infty$.

Лемма 5.2. Пусть выполнены условия теоремы 5.1. Тогда для единственного решения смешанной задачи справедлива следующая оченка:

$$
\begin{aligned}
& B\left(\left\|p_{\langle 11\rangle}, C\left(\mathbb{R}^{++}\right)\right\|+\left\|q_{1}, C\left(\mathbb{R}^{++}\right)\right\|\right) \\
& \quad \leqslant C_{0}\left(\left\|v_{b}, H^{1}\left(\mathbb{R}^{++}\right)\right\|+\left\|\varrho_{b}, H^{1}\left(\mathbb{R}^{++}\right)\right\|+\left\|T_{W}, H^{1}\left(\mathbb{R}^{++}\right)\right\|\right)
\end{aligned}
$$

т.е. смешанная задача (17) является равновесной. При $B \rightarrow \infty$ для любих $x>0$, $t \geqslant 0$ базовые компоненты решения стремятся $к$ соответствуюшим компонентам решения предельной системы уравнений Эйлера

$$
\begin{aligned}
& v(x, t, B) \rightarrow v_{\lim }(x, t)=v_{b}\left(t-\frac{x}{a_{1}}\right)+\frac{\mu_{E}}{\left(1-\mu_{E}^{2}\right)\left(\frac{3}{2} \mu_{E} \alpha^{\theta}-1\right)}\left(\frac{k}{m} T_{w}-\frac{2}{5} \varrho_{b}\right)\left(t-\frac{x}{a_{1}}\right) \\
& \varrho(x, t, B) \rightarrow \varrho_{\lim }(x, t)= v_{\lim }(x, t) \\
& T(x, t, B) \rightarrow T_{\lim }(x, t)=v_{b}\left(t-\frac{x}{a_{2}}\right)+\varrho_{b}\left(t-\frac{x}{a_{2}}\right) \\
& \quad-\frac{1+\mu_{E}}{\left(1-\mu_{E}^{2}\right)\left(\frac{3}{2} \mu_{E} \alpha^{\theta}-1\right)}\left(\frac{k}{m} T_{w}-\frac{2}{5} \varrho_{b}\right)\left(t-\frac{x}{a_{2}}\right) .
\end{aligned}
$$

Результаты леммы 5.2 следуют из длинноволнового приближения матрищы $\mathcal{R}^{\theta}$ и оценок решений смешанных задач, полученных методом перевала [5].

6. Заключение. Результаты этой статьи относятся к нелинейным системам моментов неравновесной термодинамики. Такие системы еще не изучены достаточно систематически. Мы исследовали их линеаризации в окрестности состояния равновесия и описали поведение решений задачи Коши и смешанной задачи когда параметр $B \rightarrow \infty$. Доказано, что условия глобальной устойчивости решений задачи Коши как обобщение классической теоремы Эрмита-Биллера об устойчивых полиномах; что для смешанной задачи справедлив аналог теоремы Вишика-Люстерника о мальх сингулярных возмущениях общих эллиптических задач. Последнее позволило определить условие Шапиро-Лопатинского о корректности смешанной задачи. Более того, структура общего решения начальной и смешанной задач для линеаризованных одномерных систем дает правильное разложение асимптотического решения этих задач в 2-D и 3-D случаях. Такое разложение является аналогом разложений, предложенных П. Лаксом [4].

Автор благодарен В. Дрейеру и Л.Р. Волевичу за полезные обсуждения. 


\section{СПИСОК ЦИТИРОВАННОЙ ЛИТЕРАТУРЫ}

[1] Muller I., Ruggeri T. Extended Thermodynamics: Springer-Verlag, 1993.

[2] Grad H. On the kinetic theory of rarefied gases // Comm. Pure Appl. Math. 1949. V. 2. P. 331-407.

[3] Struchtrup H., Weiss W. Temperature jump and velocity slip in the moment method // Continuum Mech. Thermodyn. 2000. V. 12. P. 1-18.

[4] Junk M. Domain of definition of Levermore's five-moment system // J. Stat. Phys. 1998. V. 93. P. $1143-1167$.

[5] Hörmander L. Lectures on Nonlinear Hyperbolic Differential Equations. Math. Appl.: Springer-Verlag, 1997.

[6] Волевич Л. Р., Радкевич Е. В. Равномерные оценки решений задачи Коши для гиперболических уравнений с малым параметром при старших производных // Дифференц. уравнения. (в печати).

[7] Hermite Ch. // Oeuvres I. Paris, 1905. P. 397-414.

[8] Babich V. M., Klimova A. A. Hyperbolic equation with large parameter at lower terms, and the hierarchy of waves // St. -Peterburg Math. J. 1995. V. 6. № 5. P. 1001-1038.

[9] Hadamard J. Sur les problemes aux derivées partielles et leur signification physique // Bull. Univ. Princeton. 1902. V. 13.

[10] Lax P. Hyperbolic system of conservation laws II // Comm. Pure Appl. Math. 1957. V. 10. P. $537-566$.

[11] Вишик М.И., Л юстерник Л. А. Асимптотическое поведение решений линейньх дифференциальных уравнений с большими или быстро меняющимися коэффициентами и граничными условиями // УМН. 1960. Т. 15. № 4. С. 27-95.

[12] Федорюк М. В. Асимптотические методы для линейных обыкновенных дифференциальных уравнений. М.: Наука, 1983. 